\title{
Photon-induced carrier transport in high efficiency mid-infrared quantum cascade lasers
}

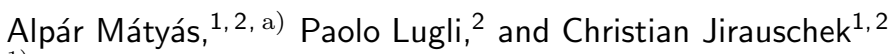 \\ 1) Emmy Noether Research Group "Modeling of Quantum Cascade Devices", Technische Universität München, \\ D-80333 Munich, Germany \\ ${ }^{2)}$ Institute for Nanoelectronics, Technische Universität München, D-80333 Munich, \\ Germany
}

(Dated: 21 March 2022, published as J. Appl. Phys. 110, 013108 (2011))

A mid-infrared quantum cascade laser with high wall-plug efficiency is analyzed by means of an ensemble Monte-Carlo method. Both the carrier transport and the cavity field dynamics are included in the simulation, offering a self-consistent approach for analyzing and optimizing the laser operation. It is shown that at low temperatures, photon emission and absorption can govern the carrier transport in such devices. Furthermore we find that photon-induced scattering can strongly affect the kinetic electron distributions within the subbands. Our results are validated against available experimental data.

\section{INTRODUCTION}

Quantum cascade lasers (QCLs) are among the most promising mid-infrared (MIR) laser sources, offering applications in gas sensing, free space communications and spectroscopy. Since the first operating device was presented in $1994, \frac{1}{1}$ QCL designs have been constantly improved with respect to their efficiency and output power. Recently QCLs with wall-plug efficiencies (WPEs) of around $50 \%$ were reported for the first time 2,3 In such structures, light emission and absorption are not only relevant with respect to the generated optical power, but also strongly affect the carrier transport in the devices. In fact, for the low temperatures where these high efficiencies are reached, the photon-induced processes dominate the other scattering mechanisms. Thus, to adequately model the operation of these lasers, the optical cavity field has to be considered in the simulation. While this is routinely done in one-dimensional simulations,,$-\underline{-7}$ the cavity field is usually neglected in fully three-dimensional (3-D) approaches like the ensem-

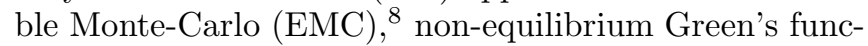
tions (NEGF) ${ }^{9,10}$ or 3-D density matrix ${ }^{11,12}$ method. However, such 3-D simulations, where the in-plane carrier dynamics is explicitly considered, do not only yield level occupations, but also the kinetic carrier distributions within these levels. Here, we employ the EMC method, which has has been intensely used to investigate the carrier transport in both $\mathrm{MIR}^{8,13}-17$ and terahertz $^{18-23}$ QCLs. To include also the optical processes, we have recently extended this approach, allowing for self-consistent coupled simulations of the carrier transport and the optical cavity field ${ }^{24}$ The EMC method is a semiclassical approach, i.e., quantum correlations are neglected in contrast to $\mathrm{NEGF}^{25}$ or density matrix ${ }^{12}$ calculations; however, the carrier transport in MIR QCLs has been shown to be largely incoherent.12

\footnotetext{
a) Electronic

mail: http://www.nano.ei.tum.de/noether
}

The goal of the present study is to analyze the carrier transport and lasing operation in a record-efficiency MIR QCL $\stackrel{3}{\underline{3}}$ with a particular focus on the influence of photoninduced scattering on the carrier transport. Specifically, we show that the inclusion of light emission and absorption in the simulation is crucial to obtain a realistic description for such devices. Furthermore, our analysis provides insight into the carrier dynamics on a microscopic level, for example the kinetic electron distributions in the upper and lower laser level which are hardly accessible to experimental observation. The paper is organized as follows: In Section II], we give a basic overview of our MonteCarlo approach, specifically adapted for InGaAs/InAlAs strain-compensated MIR QCLs. In Section III, we compare our simulation results to available experimental data and demonstrate the strong influence of stimulated emission on the carrier transport. The paper is concluded in Section IV

\section{METHOD}

The EMC method is based on the semiclassical Boltzmann transport equation. ${ }^{12}$ Scattering is self-consistently accounted for based on Fermi's golden rule. All the relevant mechanisms like electron (e)-longitudinal optical (LO) and acoustic phonon, e-interface roughness, eimpurity and e-e scattering are routinely considered in our simulation tool. $\frac{26,27}{}$ Moreover, various effects relevant for MIR QCLs based on the InGaAs/InAlAs material system have been added. We have included random alloy scattering, ${ }^{28}$ with a scattering potential of $0.3 \mathrm{eV}$ reported for high indium content InGaAs. ${ }^{29}$ Furthermore, we account for InAs- and GaAs-like phonons, using their composition dependent values for the phonon energy. 30 The scattering rates are weighted by the concentration of the individual materials (InAs and GaAs). The influence of the AlAs-like branch is believed to be negligible in QCL structures. ${ }^{31}$ Here, the bulk phonon approximation is adopted, which was shown to be a valid approach for the simulation of such QCL structures, 16 
The (parallel and perpendicular) effective masses have been implemented considering strain 32 and nonparabolicity. Our implementation of non-parabolicity is based on the approach developed by Ekenberg. 33 Nonparabolicity parameters were determined from the material bandgap, $\frac{34}{2}$ using temperature dependent values. 35 In the InGaAs material system, the parallel nonparabolicity is enhanced by a factor of 1.7 as compared to the perpendicular value. 36 The perpendicular effective mass affects the subband energies and wavefunctions, as considered in our Schrödinger-Poisson solver $\stackrel{37}{2}$ The parallel effective mass is accounted for by assigning a different value to each subband, affecting the scattering rates in the EMC solver. Here we focus on simulations at a lattice temperature of $40 \mathrm{~K}$ where the investigated structure operates with a record wall-plug efficiency of above $50 \% \frac{3}{3}$ At such low temperatures, the kinetic electron energies are still moderate, whereas for room temperature operation, a more complex implementation of nonparabolicity might be required, e.g., based on $\mathbf{k} \cdot \mathbf{p}$ theory. ${ }^{14-16}$ Furthermore, at low temperatures, the electron leakage into indirect valleys, not considered in our simulations, is very small 14,15

The interface roughness is typically described by a mean height $\Delta$ and a correlation length $\Lambda$. In contrast to the well-known bulk material parameters, this quantity is hardly accessible to experimental measurement and depends critically on the growth conditions. Thus, there is an uncertainty regarding the values of $\Delta$ and $\Lambda .26,38$ However, experimental data indicate that $\Delta \Lambda \approx 1 \mathrm{~nm}^{2}$ for the InGaAs/InAlAs structures,$\stackrel{39,40}{ }$ reducing the uncertainty to a single parameter value. We choose $\Delta=0.06 \mathrm{~nm}$, which yields the best agreement with the experimental results. This value is somewhat lower than previously used values for strain-free lattice-matched structures 39,41 However, we note that vertical correlations, which are not included in our simulations, can reduce the effect of interface roughness for strained (e.g., strain-balanced) quantum cascade lasers, $\stackrel{39}{3}$ as considered here.

Lasing is implemented based on a recently published approach, treating the photon dynamics in terms of classical intensity evolution equations and accounting for photon-induced scattering in the EMC solver ${ }^{24,42}$ In this way we can self-consistently describe the coupled carrierlight dynamics due to absorption as well as stimulated and spontaneous emission. For the investigated design operating at $5 \mu \mathrm{m}, \frac{3}{3}$ the mirror loss, which amounts to $6.4 \mathrm{~cm}^{-1}$ for a $2 \mathrm{~mm}$ long structure, dominates the waveguide loss, which is about $0.5 \mathrm{~cm}^{-1}$ for such cavities. ${ }^{43}$ The confinement factor is chosen to be 0.8 as found for a similar design. .43 For our simulation, we use 1200 longitudinal modes in the frequency range between 50 and $80 \mathrm{THz}$, corresponding to a Fabry-Perot mode spacing of $25 \mathrm{GHz}$.

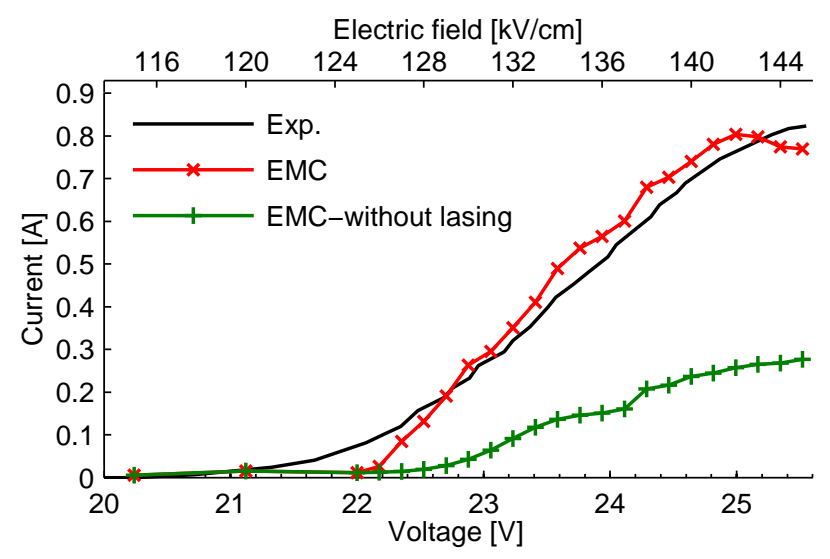

FIG. 1. (Color online) Current-voltage characteristics. The EMC simulation results with (X marks) and without (crosses) lasing included are compared to available experimental data ${ }^{3}$ (solid curve). The electric current is governed by stimulated photon emission and absorption processes, as has to be expected for a WPE as high as $50 \%$.

\section{RESULTS}

Results are presented for a recently fabricated high efficiency QCL operating at $5 \mu \mathrm{m} .3$ The simulations were performed at a lattice temperature of $40 \mathrm{~K}$, where the record WPE of $53 \%$ was observed.

In Fig. 1, we compare the current-voltage characteristics provided by EMC simulations to experiment. The simulations were performed at biases ranging from $115 \mathrm{kV} / \mathrm{cm}$ to $145 \mathrm{kV} / \mathrm{cm}$. For comparison to experiment, these were converted to the voltage points in Fig. 1, by considering 80 stages with a thickness of $22.1 \mathrm{~nm}$ each. $\underline{3}$ Above threshold, good agreement is found if lasing is included, while the current due to non-radiative processes (EMC without lasing) is lower by a factor of almost 3 than the experimentally measured current. This shows that stimulated processes become more and more important for a correct description of the carrier transport as the WPE of QCLs is improved. On the other hand, the spontaneous photon emission rates in our simulation are far too low to affect the carrier transport, which is in agreement with theoretical considerations $\stackrel{44}{4}$ The onset of the negative differential resistance (NDR) regime agrees well, occuring at $25.1 \mathrm{~V}$ for the EMC with lasing included and $25.6 \mathrm{~V}$ in the experiment. For low fields where the energy levels are not aligned, the simulation underestimates the experimentally observed current. Here, the scattering-induced transport is not efficient, and the remaining current can likely be attributed to coherent lowfield transport which is not included in the EMC simulation. ${ }^{25}$ For design optimization with respect to the WPE, the parasitic channels should be suppressed and the stimulated emission into the lasing modes maximized. Such a task can only be performed with an approach taking into account the optical cavity field.

In Fig. 2(a) and (b) we compare the simulated and ex- 


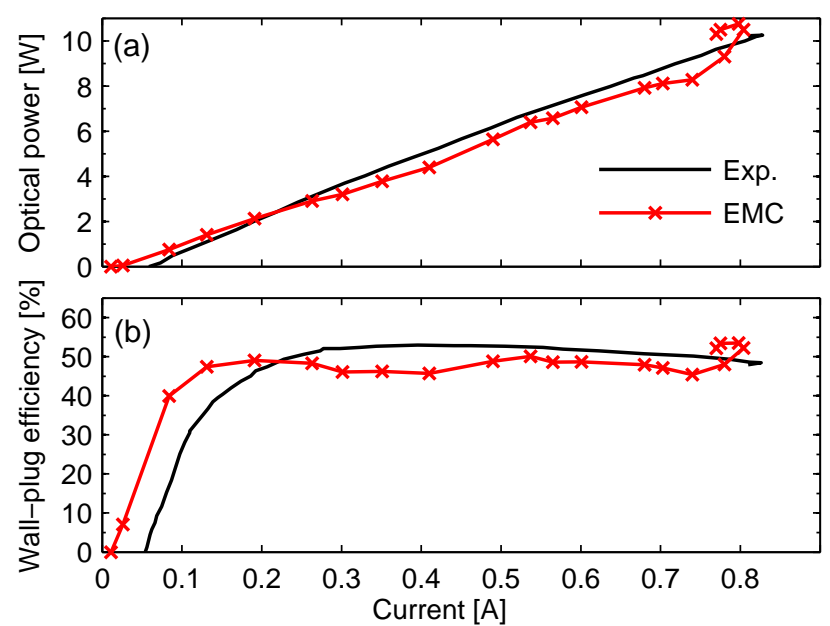

FIG. 2. (Color online) (a) Current-optical power and (b) current-WPE characteristics. The EMC simulation results with lasing included (X marks) are compared to available experimental data ${ }^{3}$ (solid curve).

perimental current-output power and current-WPE characteristics. In the EMC simulation, the bias dependent $\mathrm{WPE} \eta_{\mathrm{WPE}}$ is computed as $\eta_{\mathrm{WPE}}=P_{\mathrm{opt}} / P_{\mathrm{el}}$. Here, $P_{\text {opt }}$ is the simulated optical power emitted through both facets as in the experiment, $\underline{\underline{3}}$ and the electric power $P_{\mathrm{el}}$ is the product of the applied voltage and the simulated electric current. The simulated and experimental currentoutput power characteristics in Fig. 2(a) show excellent qualitative and quantitative agreement. The maximum emitted optical power is about $10 \mathrm{~W}$, which is in both cases obtained around the onset of NDR, where the current reaches its maximum value of $0.8 \mathrm{~A}$. For higher biases, i.e., in the NDR regime, the simulated optical power and electric current get reduced again. The simulated threshold current is lower than the experimental value, for the reasons discussed in the previous paragraph. Also the simulated and experimental current-WPE characteristics shown in Fig. 2(b) agree well. Particularly, the maximum simulated WPE of $49 \%$ below the onset of NDR compares very well to the experimental value of $53 \%$. The simulated high WPE value of $53.5 \%$ around the onset of NDR is not observed in the experiment, which we attribute to the fact that the operation in the NDR region is unstable due to domain formation. $\underline{45,46}$

Full $k$-space three-dimensional simulation approaches like EMC can yield information on the microscopic level, which is hardly accessible to experimental observation. In the following, we investigate the intrasubband kinetic carrier distributions. These can be characterized by corresponding electron temperatures only in the case of quasi-thermal equilibrium within the subbands, corresponding to a Maxwellian distribution for low doping. The kinetic electron distribution in the upper and lower laser level is shown in Fig. 3(a). The bias is $25.1 \mathrm{~V}$, where the simulated current and output power reach their max-
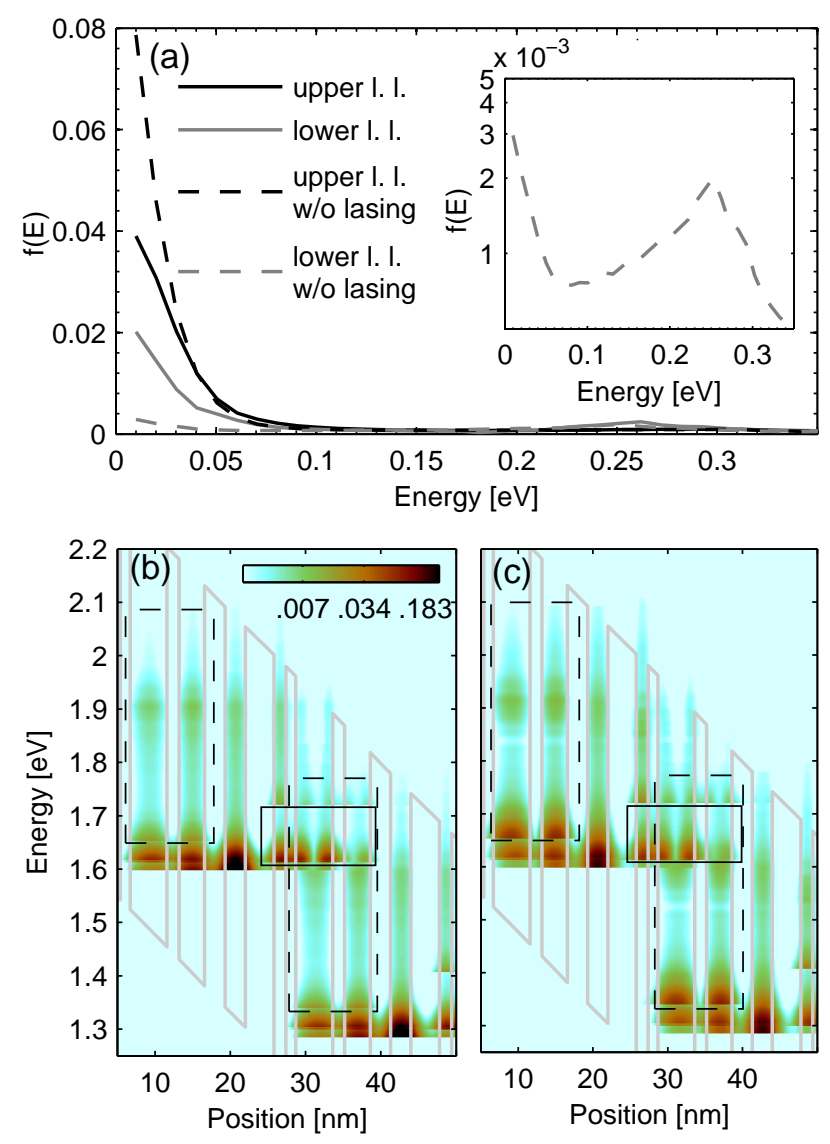

FIG. 3. (Color online) (a) Simulated kinetic electron distribution $f(E)$ in the upper and lower laser level with and without lasing included. (b) Energy resolved electron density without lasing included; (c) energy resolved electron density with lasing included. The upper laser levels are marked by solid rectangles, and the lower laser level is marked by dashed rectangles.

imum values. We note that for this bias, optical transitions from two upper levels contribute significantly to lasing. For simplicity, we restrict our discussion to one of these states, since the kinetic electron distribution function is found to be similar for the other level. The carrier distributions in the laser levels change significantly by taking lasing into account (solid curves), as compared to the case without lasing (dashed curves). The lasing action leads to a depletion of the upper laser levels and a filling of the lower laser level, corresponding to the effect of gain saturation. In the inset of Fig. 3(a), the electron distribution in the lower laser level without lasing is shown on a logarithmic scale, i.e., a Maxwellian distribution would produce a straight line. The distribution is highly non-Maxwellian with an additional peak at around $250 \mathrm{meV}$, corresponding to the energy spacing between upper and lower laser level. This bump stems from nonradiative transitions from the upper laser level, mainly LO phonon scattering as the dominant nonradia- 
tive mechanism. The energetic extension of the bump is partly due to the kinetic electron distribution in the upper laser level and the finite phonon energies of $29.5 \mathrm{meV}$ and $32.2 \mathrm{meV}$ for the two LO branches considered here. E-e scattering, which is the predominant intrasubband scattering mechanism, is unable to thermalize the intrasubband carrier distribution strongly enough to suppress the bump. ${ }^{8}, 47$ The inclusion of lasing action leads not only to a filling of the lower laser level, but also to a more thermalized kinetic electron distribution, while the bump at around $250 \mathrm{meV}$ still persists. A least square fit produces an electron temperature of $T_{\mathrm{e}}=314 \mathrm{~K}$ (upper laser level) and $T_{\mathrm{e}}=344 \mathrm{~K}$ (lower laser level) with lasing included. This is consistent with the observation that in strain compensated structures the electronic temperature is clearly above the lattice temperature. 6,48

In Fig. 33(b) and (c), the energy resolved electron density (normalized to its maximum value) is shown without and with lasing included, again for a bias of 25.1 V. For the two quantum wells located between $6 \mathrm{~nm}$ and $18 \mathrm{~nm}$, the upper laser level is omitted, so that the high-energy peak discussed in the previous paragraph (see inset of Fig. 3(a)), here located at around $1.9 \mathrm{eV}$, is clearly visible. By comparing Fig. 3(b) and (c), we can observe the changes in the energy resolved electron density for the laser levels without and with lasing included. The high-energy tails of the kinetic electron distributions remain basically unaffected. In particular, the additional high-energy peak for the lower laser level appears also if lasing is accounted for.

We have successfully tested our approach for a further high efficiency QCL design ${ }^{2}$ using the same material parameters, again finding good agreement with experiment.

\section{CONCLUSION}

Based on a self-consistent EMC carrier transport simulation including the optical cavity field, we have analyzed the effect of photon emission and absorption on the carrier transport in a high WPE quantum cascade laser. In the regime where efficient lasing is obtained, we find that the inclusion of photon-induced scattering is crucial for the correct calculation of the device current. Furthermore, a comparison to experimental data yields very good agreement for the optical output power and WPE. An analysis of those quantities, as also needed for design optimization, is only possible with an approach which includes both the carrier transport and the optical cavity field. The EMC method also enables us to investigate microscopic quantities such as the intrasubband kinetic carrier distributions, hardly accessible to experimental observation. The upper and lower laser level carrier distributions are strongly affected by the lasing action and approach each other, corresponding to gain saturation. We observe strong deviations from an equilibrium distribution especially for the lower laser level, where a highenergy peak in the electron distribution is found, caused by parasitic transitions from the upper laser level. Our results show that the chosen approach is well suited to model high efficiency MIR QCLs on a qualitative and quantitative level, and to analyze the laser operation on a microscopic scale which is hardly accessible to experimental observations.

\section{ACKNOWLEDGMENTS}

A. M. and C. J. acknowledge support from the Emmy Noether program of the German Research Foundation (DFG, JI115/1-1) . We acknowledge computational resources from the LRZ (t8481ag). A. M. additionally acknowledges support from the TUM Graduate School.

${ }^{1}$ J. Faist, F. Capasso, D. L. Sivco, C. Sirtori, A. L. Hutchinson, and A. Y. Cho, Science 264, 553 (1994).

${ }^{2}$ P. Q. Liu, A. J. Hoffman, M. D. Escarra, K. J. Franz, J. B. Khurgin, Y. Dikmelik, X. Wang, J.-Y. Fan, and C. F. Gmachl, Nat. Photonics 4, 95 (2010).

${ }^{3}$ Y. Bai, S. Slivken, S. Kuboya, S. R. Darvish, and M. Razeghi, Nat. Photonics 4, 99 (2010).

${ }^{4}$ V.-M. Gkortsas, C. Wang, L. Kuznetsova, L. Diehl, A. Gordon, C. Jirauschek, M. A. Belkin, A. Belyanin, F. Capasso, and F. X. Kärtner, Opt. Express 18, 13616 (2010).

${ }^{5}$ L. Schrottke, M. Wienold, M. Giehler, R. Hey, and H. T. Grahn, J. Appl. Phys. 108, 103108 (2010).

${ }^{6}$ R. Terazzi and J. Faist, New J. Phys. 12, 033045 (2010).

${ }^{7}$ G. Beji, Z. Ikonić, C. A. Evans, D. Indjin, and P. Harrison, J. Appl. Phys. 109, 013111 (2011).

${ }^{8}$ R. C. Iotti and F. Rossi, Appl. Phys. Lett. 78, 2902 (2001).

${ }^{9}$ F. Banit, S.-C. Lee, A. Knorr, and A. Wacker, Appl. Phys. Lett. 86, 041108 (2005).

${ }^{10}$ T. Kubis, C. Yeh, P. Vogl, A. Benz, G. Fasching, and C. Deutsch, Phys. Rev. B 79, 195323 (2009).

${ }^{11}$ C. Weber, F. Banit, S. Butscher, A. Knorr, and A. Wacker, Appl. Phys. Lett. 89, 091112 (2006).

${ }^{12}$ R. C. Iotti and F. Rossi, Phys. Rev. Lett. 87, 146603 (2001).

${ }^{13}$ F. Compagnone, A. di Carlo, and P. Lugli, Appl. Phys. Lett. 80, 920 (2002).

${ }^{14}$ X. Gao, D. Botez, and I. Knezevic, J. Appl. Phys. 101, 063101 (2007).

${ }^{15}$ X. Gao, M. D'Souza, D. Botez, and I. Knezevic, J. Appl. Phys. 102, 113107 (2007).

${ }^{16}$ X. Gao, D. Botez, and I. Knezevic, J. Appl. Phys. 103, 073101 (2008).

${ }^{17}$ P. Borowik, J.-L. Thobel, and L. Adamowicz, J. Appl. Phys. 108, 073106 (2010).

${ }^{18}$ R. Köhler, R. C. Iotti, A. Tredicucci, and F. Rossi, Appl. Phys. Lett. 79, 3920 (2001).

${ }^{19}$ H. Callebaut, S. Kumar, B. S. Williams, Q. Hu, and J. L. Reno, Appl. Phys. Lett. 83, 207 (2003).

${ }^{20}$ O. Bonno, J.-L. Thobel, and F. Dessenne, J. Appl. Phys. 97, 043702 (2005).

${ }^{21}$ C. Jirauschek, G. Scarpa, P. Lugli, M. S. Vitiello, and G. Scamarcio, J. Appl. Phys. 101, 086109 (2007).

${ }^{22}$ H. Li, J. C. Cao, Y. J. Han, X. G. Guo, Z. Y. Tan, J. T. Lü, H. Luo, S. R. Laframboise, and H. C. Liu, J. Appl. Phys. 104, 043101 (2008).

${ }^{23}$ A. Mátyás, M. A. Belkin, P. Lugli, and C. Jirauschek, Appl. Phys. Lett. 96, 201110 (2010).

${ }^{24}$ C. Jirauschek, Appl. Phys. Lett. 96, 011103 (2010).

${ }^{25}$ A. Mátyás, T. Kubis, P. Lugli, and C. Jirauschek, Physica E 42, 2628 (2010).

${ }^{26}$ C. Jirauschek and P. Lugli, J. Appl. Phys. 105, 123102 (2009). 
${ }^{27}$ C. Jirauschek, A. Mátyás, and P. Lugli, J. Appl. Phys. 107, 013104 (2010).

${ }^{28}$ T. Unuma, M. Yoshita, T. Noda, H. Sakaki, and H. Akiyama, J. Appl. Phys. 93, 1586 (2003).

${ }^{29}$ P. Ramvall, N. Carlsson, P. Omling, L. Samuelson, W. Seifert, Q. Wang, K. Ishibashi, and Y. Aoyagi, J. Appl. Phys. 84, 2112 (1998).

${ }^{30}$ A. M. Alcalde and G. Weber, J. Appl. Phys. 85, 7276 (1999).

${ }^{31}$ O. Drachenko, J. Galibert, J. Léotin, J. W. Tomm, M. P. Semtsiv, M. Ziegler, S. Dressler, U. Müller, and W. T. Masselink, Appl. Phys. Lett. 87, 072104 (2005).

${ }^{32}$ M. Sugawara, N. Okazaki, T. Fujii, and S. Yamazaki, Phys. Rev. B 48, 8102 (1993).

${ }^{33}$ U. Ekenberg, Phys. Rev. B 40, 7714 (1989).

${ }^{34}$ D. F. Nelson, R. C. Miller, and D. A. Kleinman, Phys. Rev. B 35, 7770 (1987).

${ }^{35}$ I. Vurgaftman, J. R. Meyer, and L. R. Ram-Mohan, J. Appl. Phys. 89, 5815 (2001).

${ }^{36}$ G. Hendorfer, M. Seto, H. Ruckser, W. Jantsch, M. Helm, G. Brunthaler, W. Jost, H. Obloh, K. Köhler, and D. J. As, Phys. Rev. B 48, 2328 (1993).

${ }^{37}$ C. Jirauschek, IEEE J. Quantum Elect. 45, 1059 (2009).
${ }^{38}$ T. Kubis, C. Yeh, and P. Vogl, Phys. Status Solidi C 5, 232 (2008).

${ }^{39}$ S. Tsujino, A. Borak, E. Müller, M. Scheinert, C. V. Falub, H. Sigg, D. Grützmacher, M. Giovannini, and J. Faist, Appl. Phys. Lett. 86, 062113 (2005).

${ }^{40}$ A. Wittmann, Y. Bonetti, J. Faist, E. Gini, and M. Giovannini, Appl. Phys. Lett. 93, 141103 (2008).

${ }^{41}$ A. Vasanelli, A. Leuliet, C. Sirtori, A. Wade, G. Fedorov, D. Smirnov, G. Bastard, B. Vinter, M. Giovannini, and J. Faist, Appl. Phys. Lett. 89, 172120 (2006).

${ }^{42}$ C. Jirauschek, Opt. Express 18, 25922 (2010).

${ }^{43}$ M. Razeghi, IEEE J. Sel. Top. Quant. 15, 941 (2009).

${ }^{44} \mathrm{M}$. Yamanishi, T. Edamura, K. Fujita, N. Akikusa, and H. Kan, IEEE J. Quantum Electron. 44, 12 (2008).

${ }^{45}$ A. Wacker, Appl. Phys. Lett. 97, 081105 (2010).

${ }^{46}$ M. Wienold, L. Schrottke, M. Giehler, R. Hey, and H. T. Grahn, J. Appl. Phys. 109, 073112 (2011).

${ }^{47}$ C. Jirauschek and P. Lugli, Phys. Status Solidi C 5, 221 (2008).

${ }^{48}$ M. S. Vitiello, T. Gresch, A. Lops, V. Spagnolo, G. Scamarcio, N. Hoyler, M. Giovannini, and J. Faist, Appl. Phys. Lett. 91, 161111 (2007). 